\title{
Polymorphisms in TS, MTHFR and ERCC1 genes as predictive markers in first-line platinum and pemetrexed therapy in NSCLC patients
}

\author{
Paweł Krawczyk • Tomasz Kucharczyk • Dariusz M. Kowalski • Tomasz Powrózek • Rodryg Ramlau • \\ Ewa Kalinka-Warzocha • Kinga Winiarczyk • Magdalena Knetki-Wróblewska • Kamila Wojas-Krawczyk • \\ Katarzyna Kałakucka $\cdot$ Wojciech Dyszkiewicz $\cdot$ Maciej Krzakowski $\cdot$ Janusz Milanowski
}

Received: 5 May 2014 / Accepted: 17 June 2014 / Published online: 16 July 2014

(C) The Author(s) 2014. This article is published with open access at Springerlink.com

\begin{abstract}
Purpose We presented retrospective analysis of up to five polymorphisms in TS, MTHFR and ERCCl genes as molecular predictive markers for homogeneous Caucasian, non-squamous NSCLC patients treated with pemetrexed and platinum front-line chemotherapy.

Methods The following polymorphisms in DNA isolated from 115 patients were analyzed: various number of 28-bp tandem repeats in 5'-UTR region of TS gene, single nucleotide polymorphism (SNP) within the second tandem repeat
\end{abstract}

P. Krawczyk $(\varangle) \cdot$ T. Kucharczyk · T. Powrózek ·

K. Wojas-Krawczyk $\cdot$ K. Kałakucka $\cdot$ J. Milanowski

Department of Pneumonology, Oncology and Allergology,

Medical University of Lublin, Jaczewskiego 8, 20-954 Lublin,

Poland

e-mail: krapa@poczta.onet.pl

T. Kucharczyk

Postgraduate School of Molecular Medicine, Warsaw Medical

University, Warsaw, Poland

D. M. Kowalski · K. Winiarczyk · M. Knetki-Wróblewska ·

M. Krzakowski

Department of Lung and Chest Cancer, Oncology

Centre - Institute M. Sklodowska-Curie, Warsaw, Poland

R. Ramlau $\cdot$ W. Dyszkiewicz

Wielkopolska Center of Pulmonology and Thoracosurgery

of Eugenia and Janusz Zeyland, Poznan, Poland

R. Ramlau $\cdot$ W. Dyszkiewicz

Department of Thoracic Surgery, Poznan University of Medical

Sciences, Poznan, Poland

E. Kalinka-Warzocha

Regional Centre of Oncology, Lodz, Poland

J. Milanowski

Institute of Agricultural Medicine of Lublin, Lublin, Poland of TS gene (G>C); 6-bp deletion in $3^{\prime}$-UTR region of the TS (1494de16); 677C >T SNP in MTHFR; 19007C >T SNP in ERCC1. Molecular examinations' results were correlated with disease control rate, progression-free survival (PFS) and overall survival.

Results Polymorphic tandem repeat sequence (2R, 3R) in the enhancer region of TS gene and G>C SNP within the second repeat of $3 R$ allele seem to be important for the effectiveness of platinum and pemetrexed in first-line chemotherapy. The insignificant shortening of $P F S$ in $3 R / 3 R$ homozygotes as compared to $2 \mathrm{R} / 2 \mathrm{R}$ and $2 \mathrm{R} / 3 \mathrm{R}$ genotypes were observed, while it was significantly shorter in patients carrying synchronous $3 \mathrm{R}$ allele and $\mathrm{G}$ nucleotide. The combined analysis of TS VNTR and MTHFR $677 \mathrm{C}>\mathrm{T}$ SNP revealed shortening of PFS in synchronous carriers of $3 \mathrm{R}$ allele in $T S$ and two $\mathrm{C}$ alleles in MTHFR. The strongest factors increased the risk of progression were poor PS, weight loss, anemia and synchronous presence of $3 R$ allele and $G$ nucleotide in the second repeat of $3 \mathrm{R}$ allele in TS. Moreover, lack of application of second-line chemotherapy, weight loss and poor performance status and above-mentioned genotype of $T S$ gene increased risk of early mortality.

Conclusion The examined polymorphisms should be accounted as molecular predictor factors for pemetrexedand platinum-based front-line chemotherapy in non-squamous NSCLC patients.

Keywords Non-small cell lung cancer · Pemetrexed · Platinum compounds · Gene polymorphisms

\section{Introduction}

Lung cancer is the main cause of death among all cancer patients in the world. Over $85 \%$ of such cases are 
represented by non-small cell lung cancer (NSCLC) and only $15 \%$ of those cases have a chance of surgical resection (Dela Cruz et al. 2011). This is why systemic chemotherapy and radiotherapy are the main treatment options in NSCLC.

Standard first-line chemotherapy consisting of platinum compounds and drugs like gemcitabine or vinorelbine, used in NSCLC therapy, has proven to be effective, but at a cost of sometimes serious side effects (Sculier and Moro-Sibilot 2009). Therefore, new 3rd generation drugs (pemetrexed) with lower toxicity profiles are being developed and used in combination with platinum compounds in first-line treatment of non-squamous NSCLC patients (Scagliotti et al. 2008).

Platinum compounds are the alkylating agents, which cross-link DNA in several different ways interfering with transcription and cell division. The damaged DNA elicits DNA repair mechanisms or activates apoptosis when repair proves impossible. Excision repair cross-complementing group 1 (ERCC1) is an endonuclease, which is involved in DNA repair in nucleotide excision repair mechanism (NER). High expression of ERCC1 and other NER enzymes is associated with a high yield of DNA repair. Increased DNA repair is associated with longer survival of surgically treated patients, but is negative predictive factor for chemotherapy using platinum compounds (Zheng et al. 2007; Gazdar 2007).

Pemetrexed is a novel cytostatic drug, which has shown efficacy in first and second line of treatment, as well as in maintenance therapy, of non-squamous NSCLC and malignant pleural mesothelioma (Scagliotti et al. 2008, 2009; Al-Saleh et al. 2012). Pemetrexed is a multitarget antifolate agent that inhibits enzymes involved in pyrimidine and purine synthesis. Its main target is thymidylate synthase (TS). Inhibition of TS results in decreased amount of thymidyne, which is necessary for DNA repair and synthesis. Pemetrexed also inhibits two other enzymes involved in purine/pyrimidine synthesis pathway: dihydrofolate reductase (DHFR) and glycinamide ribonucleotide formyltransferase (GARFT). It is also known that the activity of those target enzymes alters the effectiveness of pemetrexed. High expression of TS, DHFR and GARFT in squamous cell lung cancer and some of lung adenocarcinoma was proven to reduce chemosensitivity of cancer cells to pemetrexed, and pemetrexed was inefficient in patients with this histologic subtype of NSCLC (Scagliotti et al. 2009; Chattopadhyay et al. 2007; Hanauske et al. 2007; Wang et al. 2014; Ceppi et al. 2006).

All three enzymes are folate dependent, and hence, the activity of pemetrexed also depends on cell folate level. It has been shown that increased levels of extracellular folates decreased pemetrexed activity in human lung and colon cancer cell lines (Chattopadhyay et al. 2007). Enzyme that is indirectly involved in the proper functioning of purine and pyrimidine synthesis pathway is 5,10-methylenetetrahydrofolate reductase (MTHFR). It catalyzes an irreversible conversion of 5,10-methylenetetrahydrofolate (5,10-methyleneTHF) to methyltetrahydrofolate (5-methylTHF). Elevated levels of 5,10-methyleneTHF causes higher activity of TS and lower effectiveness of antifolate agents in cancers patients (Tiseo et al. 2012).

Thymidylate synthase mRNA level is regulated by three different polymorphisms: various number of 28-base-pair (bp) tandem repeats (VNTR) in 5'-UTR enhancer region of TS gene (TSER) (Mandola et al. 2003); a single nucleotide polymorphism (SNP) $\mathrm{G}>\mathrm{C}$ in the second repeat of 28-bp repeats; and a 6-bp deletion on the $3^{\prime}$ end of the TS gene (1494del6) (Kawakami and Watanabe 2003; Uchida et al. 2004; Mandola et al. 2004; Stoehlmacher et al. 2009). VNTR and 1494del6 polymorphisms of TS gene are known to affect the effectiveness of 5-fluorouracil treatment of metastatic colorectal cancer and breast cancer patients (Dotor et al. 2006; Kumar et al. 2010). A polymorphism $677 \mathrm{C}>\mathrm{T}$ in MTHFR gene was found to decrease the gene expression and hence to cause elevated levels of 5,10-methyleneTHF (Sohn et al. 2013). Several authors demonstrated that $19007 \mathrm{C}>\mathrm{T}$ (Asn118Asn) polymorphism of ERCCl gene is involved in ability to DNA repair. However, correlation between genotypes of $E R C C 1$ gene and the expression of ERCC1 protein in cancer cells is still controversial (Mlak et al. 2013).

In our retrospective, non-randomized, multicenter study, we tried to assess the usefulness of TS, MTHFR and $E R C C 1$ genes polymorphisms detected in venous blood cells as molecular predictive markers for first-line pemetrexed and platinum therapy in NSCLC patients.

\section{Materials and methods}

The studied group consisted of 115 non-squamous NSCLC patients treated with pemetrexed in combination with platinum compounds (cisplatin in 108 patients, carboplatin in 7 patients). All patients have been verified pathomorphologically and staged by computed tomography. Medical history data of each patient were collected. Patients' performance status was assessed according ECOG-WHO score. Patients were qualified for the therapy between 2008 and 2012 year. About $500 \mathrm{mg} / \mathrm{m}^{2}$ of pemetrexed was administered as an intravenous infusion on day 1 of each 21 day cycle. Cisplatin $75 \mathrm{mg} / \mathrm{m}^{2}$ infused over $2 \mathrm{~h}$ beginning approximately $30 \mathrm{~min}$ after the end of the pemetrexed administration. Carboplatin was dosed according to New Guidelines for Carboplatin Dosing. To reduce toxicity, patients were pretreated with folic acid and B12 vitamin. Clinical characteristic of patients is presented in Table 1.

Response to chemotherapy has been described according to RECIST criteria. During observation period (2008-February 2014), progression was observed in 85 patients and 57 patients died (complete observations). 
Table 1 Characteristic of studied group

\begin{tabular}{|c|c|}
\hline Factor & Characteristics \\
\hline Whole group $(n)$ & 115 \\
\hline \multicolumn{2}{|l|}{ Gender } \\
\hline Male $(n, \%)$ & $59(51.3)$ \\
\hline Female $(n, \%)$ & $56(48.7)$ \\
\hline Age in years (median $\pm \mathrm{SD}$ ) & $61 \pm 8.5$ \\
\hline$\leq 61$ years $(n, \%)$ & $50(43.5)$ \\
\hline$>61$ years $(n, \%)$ & $65(56.5)$ \\
\hline \multicolumn{2}{|l|}{ Performance status (PS) } \\
\hline $\mathrm{PS}=0(n, \%)$ & $38(33)$ \\
\hline $\mathrm{PS}=1(n, \%)$ & $77(67)$ \\
\hline \multicolumn{2}{|l|}{ Weight loss } \\
\hline$<5 \%(n, \%)$ & $61(53)$ \\
\hline$\geq 5 \%(n, \%)$ & $54(47)$ \\
\hline \multicolumn{2}{|l|}{ Anemia } \\
\hline Yes $(n, \%)$ & $42(36.5)$ \\
\hline No $(n, \%)$ & $73(63.5)$ \\
\hline \multicolumn{2}{|l|}{ Smoking status } \\
\hline Smokers $(n, \%)$ & $72(62.6)$ \\
\hline Non-smokers $(n, \%)$ & $21(18.3)$ \\
\hline Data not available $(n, \%)$ & $22(19.1)$ \\
\hline \multicolumn{2}{|l|}{ Disease stage } \\
\hline Inoperable (II-IIIB) $(n, \%)$ & $29(25.2)$ \\
\hline Advanced (IV) $(n, \%)$ & $86(74.8)$ \\
\hline \multicolumn{2}{|l|}{ Pathomorphological diagnosis } \\
\hline Adenocarcinoma $(n, \%)$ & $106(92.2)$ \\
\hline Large cell carcinoma $(n, \%)$ & $8(7)$ \\
\hline Adenosquamous cell carcinoma $(n, \%)$ & $1(0.8)$ \\
\hline \multicolumn{2}{|l|}{ Prior surgical treatment } \\
\hline Yes $(n, \%)$ & $26(22.6)$ \\
\hline No $(n, \%)$ & $89(77.4)$ \\
\hline \multicolumn{2}{|l|}{ Radiotherapy } \\
\hline Yes $(n, \%)$ & $22(19.1)$ \\
\hline No $(n, \%)$ & $93(80.9)$ \\
\hline $\begin{array}{l}\text { No. of first-line chemotherapy cycles (median, } \\
\text { range) }\end{array}$ & $4,1-9$ \\
\hline \multicolumn{2}{|l|}{ First-line chemotherapy } \\
\hline Cisplatin + pemetrexed $(n, \%)$ & $108(93.9)$ \\
\hline Carboplatin + pemetrexed $(n, \%)$ & $7(6.1)$ \\
\hline \multicolumn{2}{|l|}{ Second-line chemotherapy } \\
\hline Docetaxel $(n, \%)$ & $10(8.7)$ \\
\hline Paclitaxel $(n, \%)$ & $8(7)$ \\
\hline Erlotinib $(n, \%)$ & $4(3.5)$ \\
\hline Crizotinib $(n, \%)$ & $2(1.7)$ \\
\hline Belagenpumatucel-L $(n, \%)$ & $2(1.7)$ \\
\hline Vinorelbine $(n, \%)$ & $5(4.3)$ \\
\hline Carboplatin + vinorelbine $(n, \%)$ & $1(0.9)$ \\
\hline Gemcitabine $(n, \%)$ & $4(3.5)$ \\
\hline Cisplatin + gemcitabine $(n, \%)$ & $2(1.7)$ \\
\hline Pemetrexed $(n, \%)$ & $3(2.6)$ \\
\hline None $(n, \%)$ & $74(64.3)$ \\
\hline
\end{tabular}

Table 1 continued

\begin{tabular}{ll}
\hline Factor & Characteristics \\
\hline Third-line therapy & \\
Docetaxel $(n)$ & 1 \\
Gemcitabine $(n)$ & 1 \\
Eribulin $(n)$ & 1 \\
Erlotinib $(n)$ & 1 \\
\hline
\end{tabular}

Blood samples of examined patients have been collected in four oncology centers in Poland. All molecular examinations have been performed in one clinical laboratory in Pneumonology, Oncology and Allergology Department in Lublin. Five milliliters of blood samples have been collected in EDTA-covered tubes. DNA was extracted from peripheral blood cells using QIAamp DNA Mini Kit (Qiagen, Germany). This study has been approved by the Ethics Committee of Medical University in Lublin (No. KE-0254/219/2010).

\section{$T S$ and MTHFR genotyping}

TS gene VNTR polymorphism was analyzed by polymerase chain reaction (PCR) using primers previously described by Iacopetta et al. (2001) with further modification. The 6-bp deletion on $3^{\prime}$ end of the TS gene and the SNP $677 \mathrm{C}>\mathrm{T}$ in MTHFR gene were analyzed with PCR amplification followed by restriction fragment length polymorphism analysis (RFLP). Primers for 1494del6 were previously described by Dotor et al. (2006), whereas MTHFR primers were described by Frosst et al. (1995). Restriction enzymes used in RFLP reaction were Fermentas FastDigest DraI (Thermo Scientific, USA) for TS 1494del6 polymorphism and Fermentas FastDigest HinfI (Thermo Scientific, USA) for MTHFR $677 \mathrm{C}>\mathrm{T}$ SNP.

The polymorphism of TS (TSER) VNTR differs according to the number of 28-bp repeats and in Caucasian population is most often represented by three genotypes: two repeats/two repeats $(2 R / 2 R)$, three repeats/three repeats $(3 R / 3 R)$ and heterozygous of $2 R / 3 R$.

All PCR reaction products were later visualized on $2 \%$ agarose gel with ethidium bromide.

Additionally, Sanger sequencing was used for the validation of the presence of different polymorphic forms of examined genes.

\section{ERCCl genotyping}

ERCC1 SNP (19007C $>$ T) was detected using High Resolution Melt (HRM) method. Amplification of examined region and HRM procedure were performed in 48-well plates using the Eco Real-Time PCR device (Illumina). 
PCR cycling and HRM conditions were performed according to Precision ${ }^{\mathrm{TM}}$ HRM protocol. Before PCR reaction, all examined samples were diluted to the same concentration of DNA $(20 \mathrm{ng} / \mu \mathrm{l})$ for similar amplification of investigated gene region and improved quality of HRM. Additionally, DNA samples with all known allelic variants of 19000C $>$ T were enrolled as HRM controls. EcoStudy Software (Illumina) was used for melt curves analysis. Different genotypes of ERCCl were distinguished according to normalization data derived from the raw data plots and difference graph derived from the normalization data. HRM results of ERCC1 SNP analysis was validated by SNaPshot technique.

\section{Statistical analysis}

Chi square test was used to compare the quantity of patients with different response to treatment depending on the prevalence of clinical and genetic factors. To compare the probability of progression-free survival and overall survival between the groups with different clinical and molecular factors, the Kaplan-Meier method was used. Cox regression model with step-by-step selection was used to determine the influence of these factors on progression-free survival (PFS) and overall survival (OS) of patients treated with platinum and pemetrexed therapy.

\section{Results}

The enhancer region of TS gene (TSER) contains a polymorphic tandem repeat sequence (in our study only 2 or 3 repeats, $2 \mathrm{R}$ or $3 \mathrm{R}$ ). $22.6 \%$ of non-squamous NSCLC patients carried 2R/2R genotype, while $58.3 \%$ of patients were heterozygotes $2 \mathrm{R} / 3 \mathrm{R}$, and $19.1 \%$ of patients were homozygotes $3 \mathrm{R} / 3 \mathrm{R}$. Guanine nucleotide within the second repeat of the $3 \mathrm{R}$ alleles was found in $34.8 \%$ of examined patients ( $\mathrm{G}>\mathrm{C}$ polymorphism). Deletion of $6 \mathrm{bp}$ in 1494 position in both alleles of $T S$ gene was described in $7 \%$ of patients, but this deletion in only one allele of $T S$ gene-in $37.4 \%$ of patients. Rarer $\mathrm{T}$ allele in $677 \mathrm{C}>\mathrm{T}$ MTHFR gene polymorphism was observed in $53.9 \%$ of patients. In examined group, there were $15.6 \%$ of $\mathrm{C} / \mathrm{C}$ genotype carriers, $46.1 \%$ of C/T genotype and $38.3 \%$ of TT genotype of ERCCI gene $19007 \mathrm{C}>\mathrm{T}$ polymorphism.

The partial remission to first-line chemotherapy with platinum compounds and pemetrexed was observed in 29 patients with non-squamous NSCLC (25.2\%). Stable disease was noted in 66 of examined patients $(57.4 \%)$ and disease progression in 20 patients $(17.4 \%)$. Disease control rate was noted in $82.6 \%$ of patients. For whole group of patients, the median time of progression-free survival was 7 months, while the median time of overall survival was 14 months.

Some common clinical factors strongly affected the course of non-squamous NSCLC in patients treated with platinum compounds and pemetrexed in first-line therapy. Patients in poor performance status had significantly higher incidence of early progression during first 2 month of treatment than patients with good PS. PFS was significantly shorter in patients with anemia, body mass loss and in poor performance status as compared to patients without these factors. Regarding the overall survival of patients treated with platinum and pemetrexed, poor performance status, weight loss, anemia and lack of second-line treatment had a significant negative prognostic value. Stage of disease, gender and age had no impact on prognosis in our patients (Table 2).

Several polymorphic variants of TS and MTHFR genes were important as a predictor of platinum compounds and pemetrexed therapy effectiveness in non-squamous NSCLC patients. Polymorphic tandem repeat sequence $(2 \mathrm{R}, 3 \mathrm{R})$ in the enhancer region of TS gene (TSER) and a single nucleotide polymorphism $(\mathrm{G}>\mathrm{C})$ within the second repeat of the $3 R$ allele seem to be important for the effectiveness of this first-line chemotherapy. We observed insignificant shortening of PFS in $3 R / 3 R$ homozygotes as compared to $2 R / 2 R$ homozygotes and $2 R / 3 R$ heterozygotes. PFS was significantly shorter (6 months) in patients carrying synchronous $3 R$ allele and $G$ nucleotide in the second repeat of $3 R$ allele than in carriers of other genotypes of TS gene (Fig. 1).

Early disease progression was observed significantly more frequent in patients with $\mathrm{T} / \mathrm{T}$ genotype than in patients with $\mathrm{C} / \mathrm{C}$ or $\mathrm{C} / \mathrm{T}$ genotypes of MTHFR $677 \mathrm{C}>\mathrm{T}$ polymorphism. PFS and OS were not affected by MTHFR gene polymorphism. However, combined analysis of $T S$ VNTR polymorphism and MTHFR $677 \mathrm{C}>\mathrm{T}$ SNP revealed shortening of PSF (5 months) in synchronous carriers of 3R allele in TS gene and two $\mathrm{C}$ alleles in MTHFR gene (Table 3, Fig. 2).

Multivariate Cox logistic regression confirmed that the strongest factors that increased the risk of progression in non-squamous NSCLC patients treated with platinum and pemetrexed first-line therapy were poor performance status, weight loss, anemia and synchronous presence of $3 \mathrm{R}$ allele and $\mathrm{G}$ nucleotide in the second repeat of $3 \mathrm{R}$ allele in $T S$ gene. Moreover, clinical prognostic factors: lack of application of second-line chemotherapy, weight loss and poor performance status as well as the above-mentioned genotype of $T S$ gene increased risk of early mortality (Table 4).

\section{Discussion}

Pemetrexed with combination of platinum compounds was registered to front-line treatment of non-squamous NSCLC 


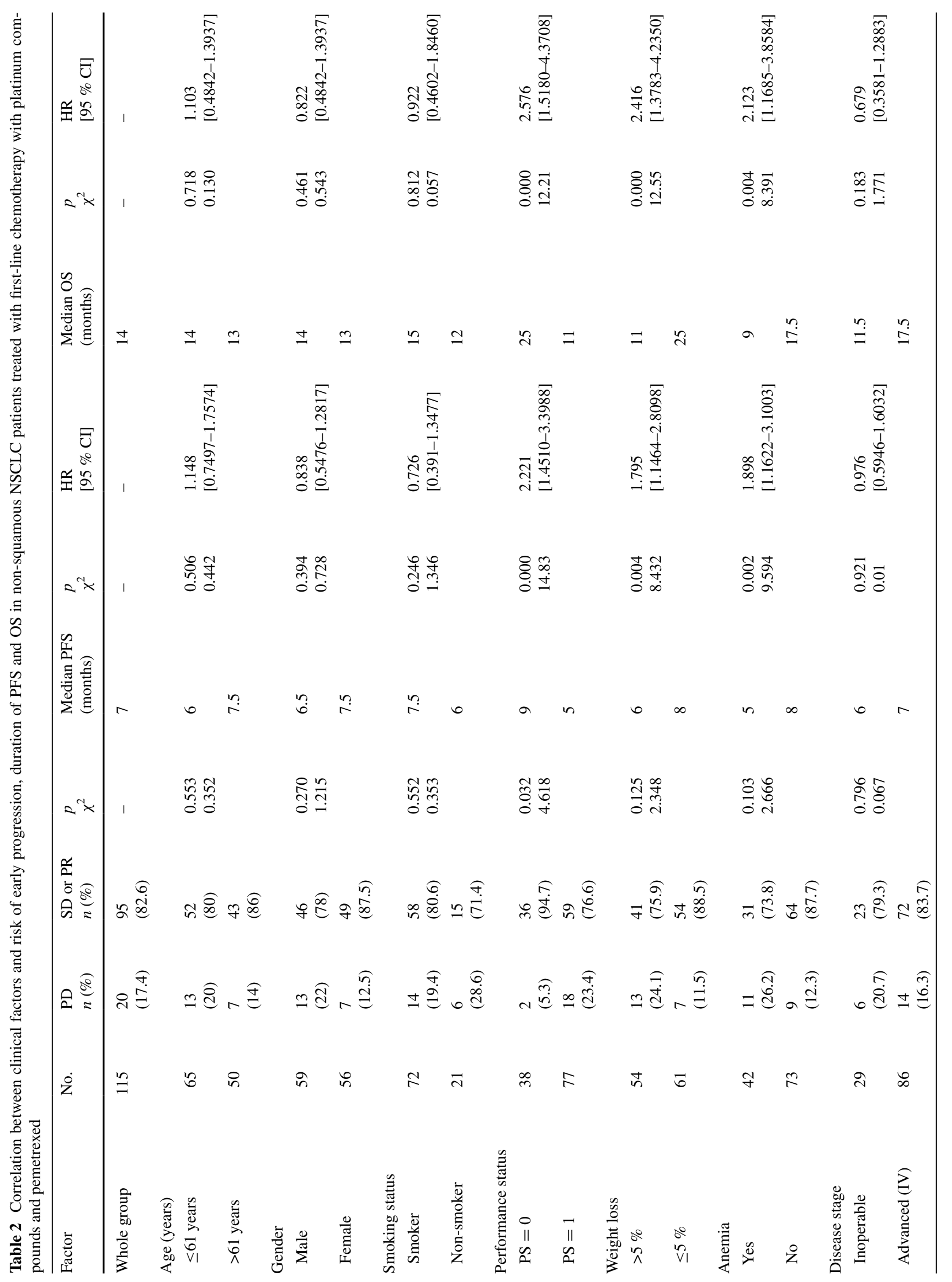




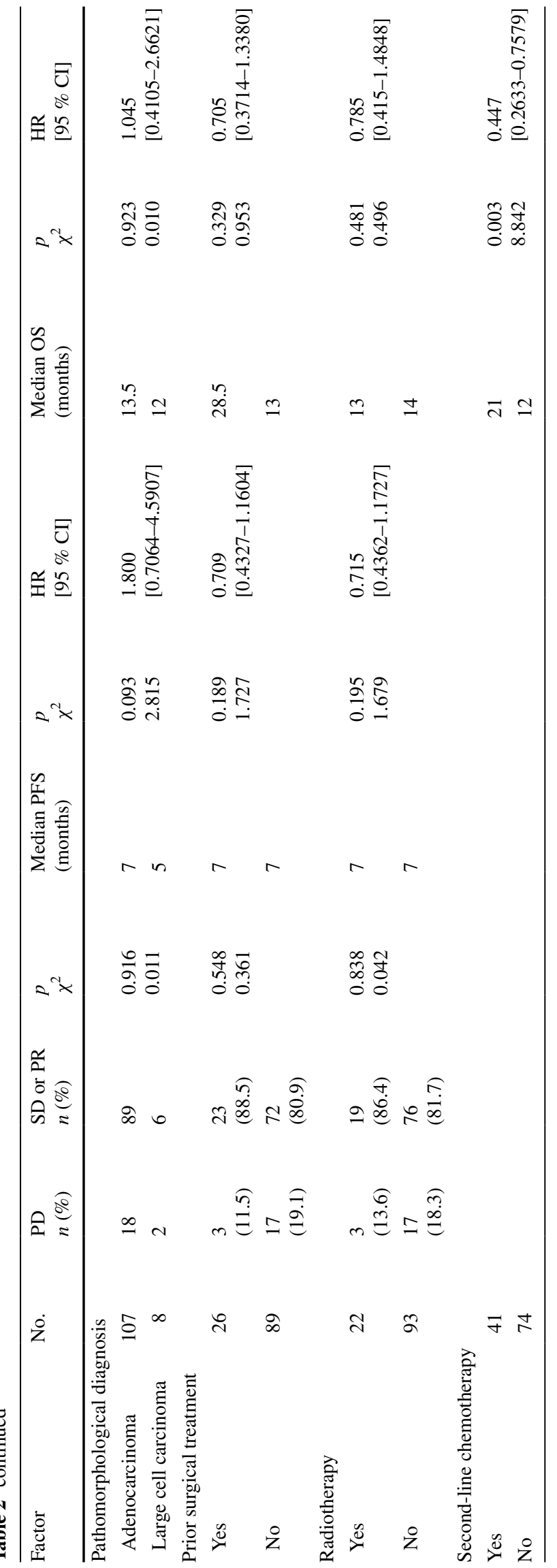

patients based on the large phase III clinical trial (Scagliotti et al. 2008), where patients randomly received either cisplatin plus gemcitabine $(n=862)$ or cisplatin plus pemetrexed ( $n=863)$. Higher response rate occurred in the cisplatin plus pemetrexed arm $(28.6 \%)$ than in cisplatin plus gemcitabine arm $(22.2 \%)$ in patients with non-squamous NSCLC. These patients had a longer OS time and trend for a longer PFS time on cisplatin plus pemetrexed (11 and 5.26 months, respectively) than on cisplatin plus gemcitabine (10.1 and 4.96 months, respectively). However, within the squamous lung cancer patients, the response rate and HR for OS as well as PFS favored cisplatin plus gemcitabine over cisplatin plus pemetrexed (Scagliotti et al. 2008, 2009, 2011).

Compared to results of Scagliotti et al., our study showed similar response rate $(25.2 \%)$ on platinum compounds and pemetrexed in non-squamous NSCLC patients. However, the duration of PFS (7 months) and OS (14 months) in our study was slightly longer than in Scagliotti et al. study. In phase III clinical trial, current and former smokers and squamous cell lung cancer patients had significantly higher risk of death compared with neversmoker and non-squamous NSCLC patients treated with cisplatin and pemetrexed. Moreover, results of phase II and III clinical trials with pemetrexed indicated factors that had statistically significant prognostic impact on survival, including gender, race, performance status and stage of disease (Scagliotti et al. 2008, 2009, 2011). In our study in Cox-adjusted analysis, we found several typical clinical factors: poor performance status, weight loss and anemia, which had affected progression and death risk. In this respect, our results were in keeping with the results

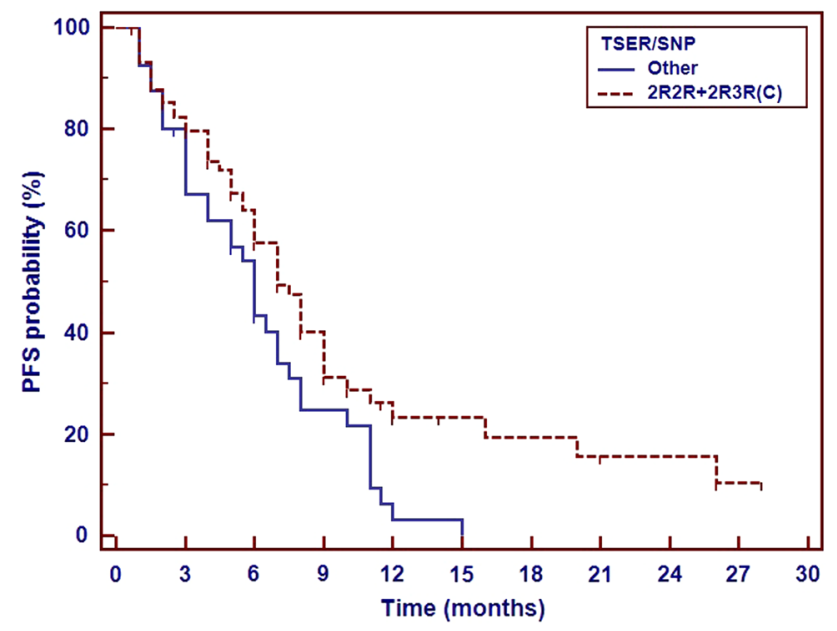

Fig. 1 Impact of TS VNTR polymorphism and a single-nucleotide polymorphism $(\mathrm{G}>\mathrm{C})$ within the second repeat of the $3 \mathrm{R}$ allele on the progression-free survival in non-squamous NSCLC patients treated with platinum and pemetrexed first-line therapy 
J Cancer Res Clin Oncol (2014) 140:2047-2057

2053

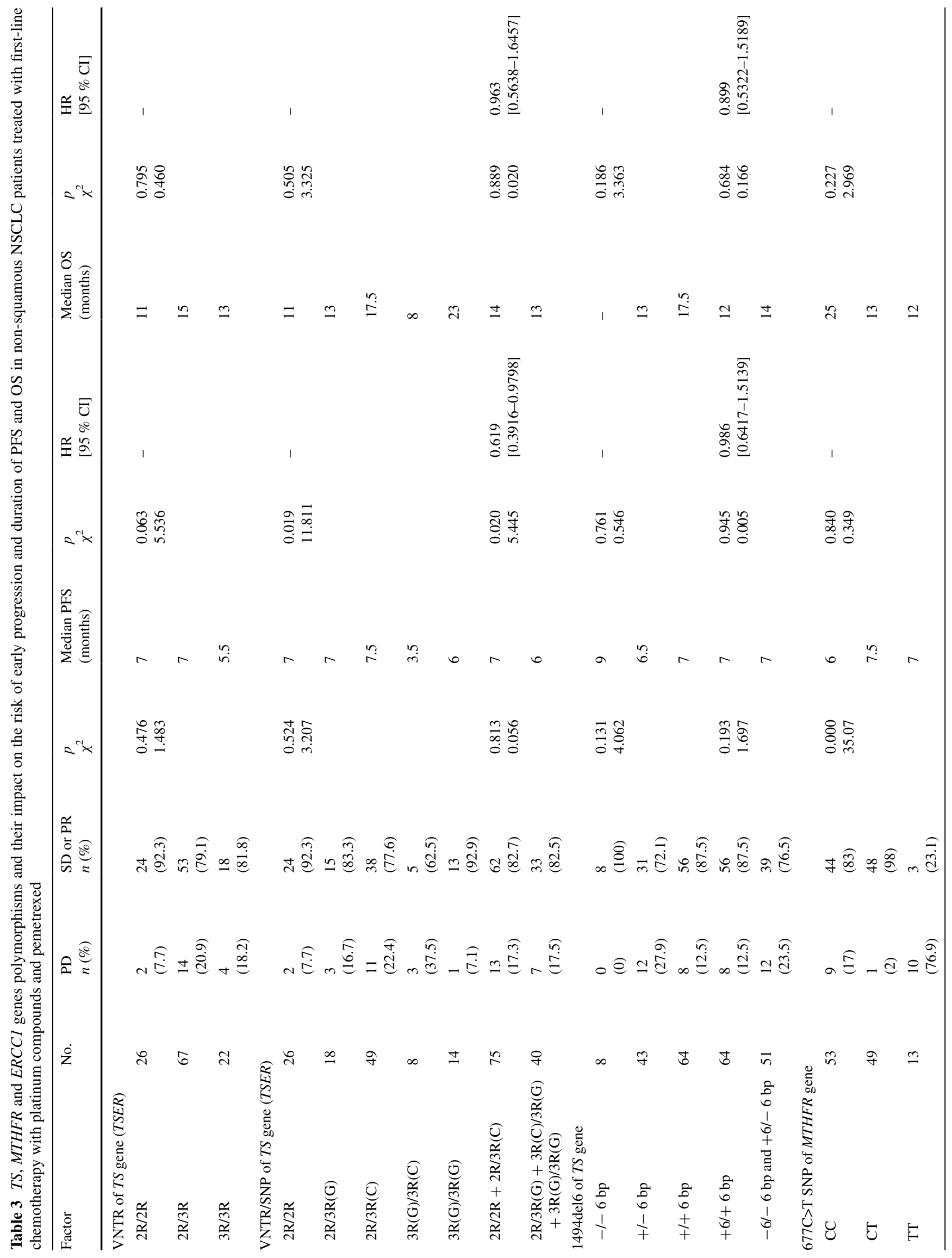

Springer 


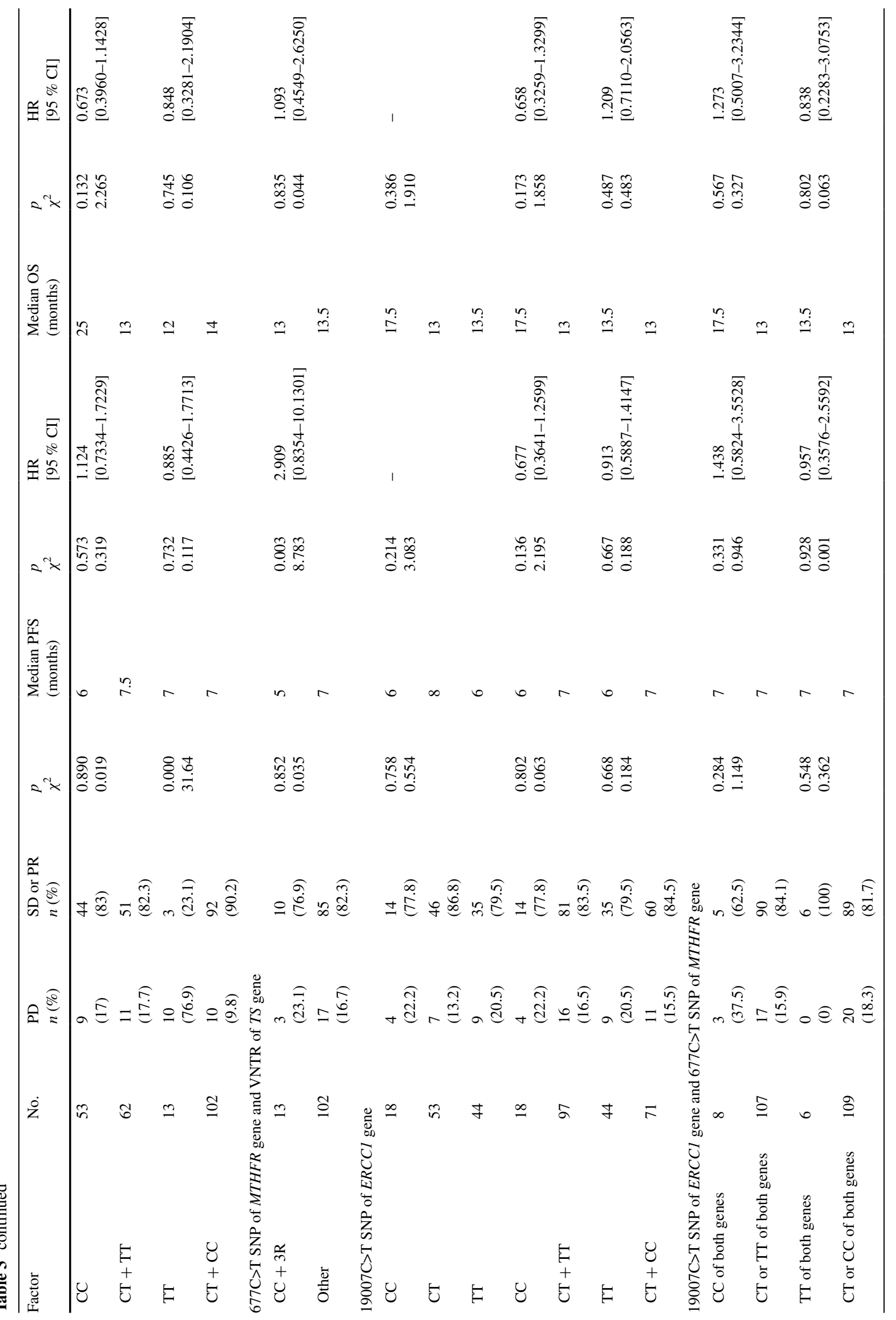


of clinical trials concerning pemetrexed therapy. However, we did not observe the differences of death risk with respect to age, smoking status, gender, stage of disease and pathomorphological diagnose (adenocarcinoma vs large cell carcinoma). The impact of post study therapy on survival is difficult to evaluate because all studies permitted subsequently treatment at the discretion of the oncologists (Scagliotti et al. 2009). However, $35.7 \%$ of our platinum plus pemetrexed-treated patients received an additional line of therapy, which resulted in prolongation of survival time to 21 months. We have to remember that these patients were in a better performance status than patients without possibility of second-line treatment.

Although the effectiveness of chemotherapy, there was a group of patients with early progression on platinum plus pemetrexed treatment (17.4\% in our study). Clinical

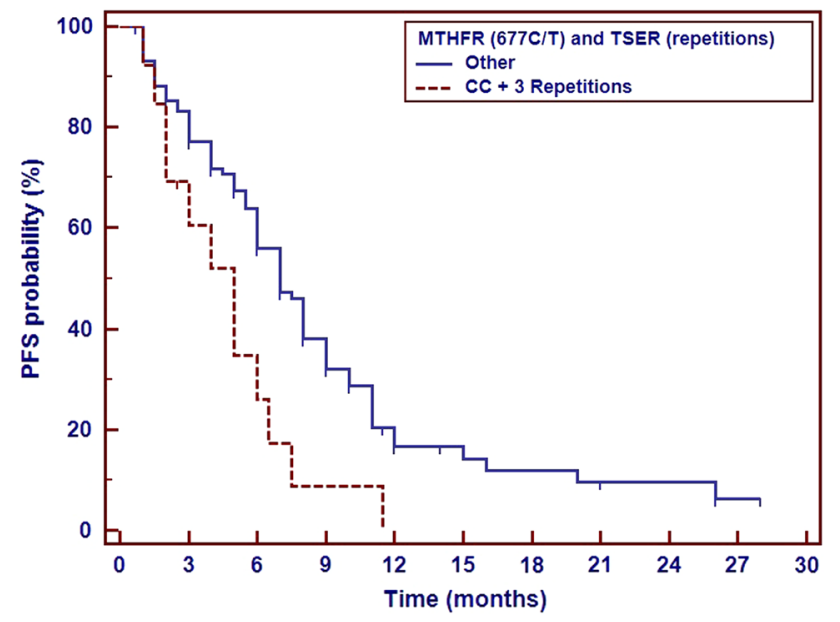

Fig. 2 Impact of TS VNTR polymorphism and MTHFR gene $677 \mathrm{C}>\mathrm{T}$ polymorphism on the progression-free survival in non-squamous NSCLC patients treated with platinum and pemetrexed firstline therapy factors are usually failed for precise prediction of treatment benefit. As for today, there are no well-defined molecular markers that are used in qualification to platinum and pemetrexed therapy in lung cancer patients. One of the best known is the relationship between the expression of ERCC1, TS, DHFR, GARFT, MTHFR as well as ERCC1 enzymes and the effectiveness of platinum and pemetrexed chemotherapy (Lee et al. 2013; Bukhari and Goudar 2013). However, the impact of the polymorphisms of genes encoding these enzymes in the efficacy of such chemotherapy is poorly described in NSCLC patients.

Li et al. (2013) had examined 45 advanced adenocarcinoma patients treated with cisplatin and pemetrexed. Median PFS was longer (6.8 vs 3.8 months) for carriers of $T S$ genotypes, leading to TS expression reduction $(2 \mathrm{R} / 2 \mathrm{R}$, $2 R / 3 R(C)$ or $3 R C / 3 R(C)$ in contrast to the patients with genotypes related to the high TS expression $(2 R / 3 R(G)$, $3 \mathrm{R}(\mathrm{C}) / 3 \mathrm{R}(\mathrm{G})$ or $3 \mathrm{R}(\mathrm{G}) / 3 \mathrm{R}(\mathrm{G})$. This $T S$ gene polymorphism had predicted the response rate. However, no difference in OS was observed (10.3 vs 10.1 months, respectively). Moreover, the PFS and OS did not differ between the patients with different MTHFR genotypes.

In Hu et al. (2012) study, 90 advance Asian lung cancer patients received pemetrexed with platinum regimens as first-line treatment or a single-agent treatment in second line or further. The variable number of tandem repeat in $5^{\prime}$-UTR region of $T S$ gene and deletion/insertion polymorphism in $3^{\prime}$-UTR region of $T S$ gene was analyzed. Disease control rate, objective response rate and PFS were similar between patients harboring $2 \mathrm{R}$ and $3 \mathrm{R}$ alleles as well as alleles containing 6-bp deletion or insertion. The authors did not observe any combined effect of both TS polymorphisms on clinical outcome of patients treated with pemetrexed-based therapy (Hu et al. 2012).

Wang et al. (2013) revealed that Chinese patients with the deletion in both $3^{\prime}$-UTR regions of TS gene (-/-6 bp) had significantly longer PFS and OS time than patients
Table 4 The multivariate Cox logistic regression of factors affecting the progression-free survival and overall survival of non-squamous NSCLC patients treated with platinum compounds and pemetrexed in first-line chemotherapy

\begin{tabular}{llll}
\hline Factor & $\beta$ & $p$ value & Hazard ratio [95\% CI] \\
\hline Progression-free survival & & & \\
$\quad$ Poor performance status & 0.8504 & 0.0008 & 2.3405 [1.4249-3.8444] \\
Lack of radiotherapy & 0.6185 & 0.0361 & $1.8561[1.0439-3.3004]$ \\
2R/3R(G) or 3R(C)/3R(G) or 3R(G)/3R(G) of $T S$ genotype & 0.5043 & 0.0249 & $1.6558[1.0681-2.5668]$ \\
Anemia & 0.4731 & 0.046 & $1.6049[1.0108-2.5482]$ \\
Overall model fit: $p<0.0001 ; \chi^{2}=26,632$ & & & \\
Overall survival & & & \\
Lack of second-line treatment & 1.1864 & 0.0008 & $3.2752[1.6421-6.5323]$ \\
2R/3R(G) or 3R(C)/3R $(\mathrm{G})$ or 3R(G)/3R(G) of $T S$ genotype & 0.7273 & 0.022 & $2.0696[1.1141-3.8445]$ \\
Weight loss $<5 \%$ & 0.7257 & 0.0153 & 2.0662 [1.1525-3.7042] \\
Poor performance status & 0.6534 & 0.0468 & $1.9221[1.0126-3.6486]$ \\
Overall model fit: $p<0.0001 ; \chi^{2}=29,974$ & & & \\
\hline
\end{tabular}


with insertion at least in one of $3^{\prime}$-UTR region $(-/+6 \mathrm{bp})$ after short-term pemetrexed treatment (Wang et al. 2013).

In contrast to above cited studies, Arvealo et al. (Arévalo et al. 2014) had examined only 25 Caucasian patients treated with pemetrexed-based regimens. Authors showed that the presence of $3 \mathrm{R} / 3 \mathrm{R}$ genotype significantly correlated with a superior response rate compared to presence of $2 R / 2 R, 2 R / 3 R$ and $3 R / 4 R$ genotypes. Moreover, a trend toward a better PFS and significant superior OS was found among subjects showing 3R/3R genotype. Active or former smokers who were homozygous for insertion $(+/+6 \mathrm{bp})$ in 3'-UTR TS region and had higher expression of TS, significantly more frequent responded to treatment than patients who were heterozygous $+/+6$ bp (Arévalo et al. 2014).

Polymorphisms of TS (both $5^{\prime}$-UTR VNTR and G>C polymorphism within the third VNTR) and MTHFR genes were tested in large study (127 Caucasian patients with complete gene polymorphisms analysis), which evaluated the effectiveness of pemetrexed plus carboplatin or pemetrexed chemotherapy. PFS was not different for patients with different $T S$ genotypes. However, carriers of TT genotype in MTHFR gene had longer PFS time compared with patients with CC or CT genotypes of this gene (Smit et al. 2009).

Our multicenter study is one of the largest involving a homogeneous group of Caucasian, non-squamous NSCLC patients treated with front-line chemotherapy based on pemetrexed and platinum and who had simultaneous analysis of up to five different polymorphisms in TS, MTHFR and $E R C C l$ genes. It should be noted that "high-expression" genotype of $T S$ gene $(2 \mathrm{R} / 3 \mathrm{R}(\mathrm{G}), 3 \mathrm{R}(\mathrm{C}) / 3 \mathrm{R}(\mathrm{G})$ or $3 R(G) / 3 R(G)$ in opposition to "low-expression" genotype of this gene $(2 R / 2 R, 2 R / 3 R(C)$ or $3 R C / 3 R(C)$ had strong negative predictive value for NSCLC patients treated with platinum compounds and pemetrexed. The predictive role of MTHFR gene polymorphism was not clear (carriers of TT genotype of MTHFR gene significantly more frequently responded to chemotherapy). However, according to our results and results of Smit et al. (2009), co-occurrence of CC genotype of MTHFR gene and 3R allele in 5'-UTR region of $T S$ gene could be assumed as negative predictive factor for pemetrexed-based chemotherapy. Whereas, $E R C C 1$ gene polymorphism may not predict the effectiveness of this chemotherapy. Moreover, the data concerning relation between the ERCC1 expression and/or ERCC1 SNP and the effectiveness of chemotherapy are debatable (Friboulet et al. 2013). Therefore, the predictive value of ERCC1 SNP for platinum-based therapy is not so obvious. It appears that the gene polymorphisms, whose expression could affect the pemetrexed' effectiveness, are stronger predictors for this type of treatment.

Unfortunately, clinical application of polymorphism analysis in qualification to platinum and pemetrexed chemotherapy has limitation as a lack of prospective clinical trials results (e.g., JMEN). Moreover, these prospective clinical trials should contain more than one chemotherapy arms, e.g., comparison of platinum and gemcitabine chemotherapy with platinum and pemetrexed administrated according to gene signature.

\section{Conflict of interest None declared}

Open Access This article is distributed under the terms of the Creative Commons Attribution License which permits any use, distribution, and reproduction in any medium, provided the original author(s) and the source are credited.

\section{References}

Al-Saleh K, Quinton C, Ellis PM (2012) Role of pemetrexed in advanced non-small-cell lung cancer: meta-analysis of randomized controlled trials, with histology subgroup analysis. Curr Oncol 19(1):e9-e15

Arévalo E, Castañón E, López I et al (2014) Thymidylate synthase polymorphisms in genomic DNA as clinical outcome predictors in a European population of advanced non-small cell lung cancer patients receiving pemetrexed. J Transl Med 12(1):98

Bukhari AA, Goudar RK (2013) Thymidylate synthase as predictive biomarker for pemetrexed response in NSCLC. Lung Cancer Inter 436409:1-7

Ceppi P, Volante M, Saviozzi S et al (2006) Squamous cell carcinoma of the lung compared with other histotypes shows higher messenger RNA and protein levels for thymidylate synthase. Cancer 107(7):1589-1596

Chattopadhyay S, Moran RG, Goldman D (2007) Pemetrexed: biochemical and cellular pharmacology. Mol Cancer Ther 6(2):404-417

Dela Cruz CS, Tanoue LT, Matthay RA (2011) Lung cancer: epidemiology, etiology, and prevention. Clin Chest Med 32:605-644

Dotor E, Cuatrecases M, Martínez-Iniesta M et al (2006) Tumor thymidylate synthase 1494del6 genotype as a prognostic factor in colorectal cancer patients receiving fluorouracil-based adjuvant treatment. J Clin Oncol 24(10):1603-1611

Friboulet L, Olaussen KA, Pignon JP et al (2013) ERCC1 isoform expression and DNA repair in non-small-cell lung cancer. N Engl J Med 368:1101-1110

Frosst P, Blom HJ, Milos R et al (1995) A candidate genetic risk factor for vascular disease: a common mutation in methylenetetrahydrofolate reductase. Nat Genet 10(1):111-113

Gazdar AF (2007) DNA repair and survival in lung cancer-the two faces of Janus. N Engl J Med 356(8):771-773

Hanauske AR, Eismann U, Oberschmidt O et al (2007) In vitro chemosensitivity of freshly explanted tumor cells to pemetrexed is correlated with target gene expression. Invest New Drugs $25: 417-423$

$\mathrm{Hu}$ Q, Li X, Su C et al (2012) Correlation between thymidylate synthase gene polymorphisms and efficacy of pemetrexed in advanced non-small cell lung cancer. Exp Ther Med 4(6):1010-1016

Iacopetta B, Grieu F, Joseph D, Elsaleh H (2001) A polymorphism in the enhancer region of the thymidylate synthase promoter influences the survival of colorectal cancer patients treated with 5-fluorouracil. Br J Cancer 85(6):827-830

Kawakami K, Watanabe G (2003) Identification and functional analysis of single nucleotide polymorphism in the 
tandem repeat sequence of thymidylate synthase gene. Cancer Res 63:6004-6007

Kumar K, Vamsy M, Jamil K (2010) Thymidylate synthase gene polymorphisms effecting 5-FU response in breast cancer patients. Cancer Biomark 6(2):83-93

Lee SH, Noh KB, Lee JS et al (2013) Thymidylate synthase and ERCC1 as predictive markers in patients with pulmonary adenocarcinoma treated with pemetrexed and cisplatin. Lung Cancer 81(1):102-108

Li WJ, Jiang H, Fang XJ et al (2013) Polymorphisms in thymidylate syntase and reduced folate carrier (SLC19A1) genes predict survival outcome in advanced non-small cell lung cancer patients treated with pemetrexed-based chemotherapy. Oncol Lett 5(4):1165-1170

Mandola MV, Stoehlmacher J, Muller-Weeks S et al (2003) A novel single nucleotide polymorphism within the $5^{\prime}$ tandem repeat polymorphism of the thymidylate synthase gene abolishes USF-1 binding and alters transcriptional activity. Cancer Res 63(11):2898-2904

Mandola MV, Stoehlmacher J, Zhang W et al (2004) A 6 bp polymorphism in the thymidylate synthase gene causes message instability and is associated with decreased intratumoral TS mRNA levels. Pharmacogenetics 14:319-327

Mlak R, Krawczyk P, Ramlau R et al (2013) Predictive value of ERCC1 and RRM1 gene single-nucleotide polymorphisms for first-line platinum- and gemcitabine-based chemotherapy in nonsmall cell lung cancer patients. Oncol Rep 30(5):2385-2398

Scagliotti GV, Parikh P, von Pawel J et al (2008) Phase III study comparing cisplatin plus gemcitabine with cisplatin plus pemetrexed in chemotherapy-naive patients with advanced-stage non-smallcell lung cancer. J Clin Oncol 26:3543-3551

Scagliotti G, Hanna N, Fossella F et al (2009) The differential efficacy of pemetrexed according to NSCLC histology: a review of two phase III studies. Oncologist 14(3):253-263

Scagliotti G, Brodowicz T, Shepherd FA et al (2011) Treatment-byhistology interaction analyses in three phase III trials show superiority of pemetrexed in nonsquamous non-small cell lung cancer. J Thorac Oncol 6:64-70
Sculier JP, Moro-Sibilot D (2009) First- and second-line therapy for advanced non-small cell lung cancer. Eur Respir J 33:915-930

Smit EF, Burgers SA, Biesma B et al (2009) Randomized phase II and pharmacogenetic study of pemetrexed compared with pemetrexed plus carboplatin in pretreated patients with advanced non-smallcell lung cancer. J Clin Oncol 27(12):2038-2045

Sohn KJ, Croxford R, Yates Z, Lucock M, Kim YI (2013) Effect of the methylenetetrahydrofolate reductase C677T polymorphism on chemosensitivity of colon and breast cancer cells to 5-fluorouracil and methotrexate. J Natl Cancer Inst 96(2):134-144

Stoehlmacher J, Goekkurt E, Hoeffken G et al (2009) Thymidylate synthase polymorphisms and immunohistochemistry in nonsmall cell lung cancer. J Clin Oncol 27(suppl):11101

Tiseo M, Giovannetti E, Tibaldi C et al (2012) Pharmacogenetic study of patients with advanced non-small cell lung cancer (NSCLC) treated with second-line pemetrexed or pemetrexed-carboplatin. Lung Cancer 78(1):92-99

Uchida K, Hayashi K, Kawakami K et al (2004) Loss of heterozygosity at the thymidylate synthase (TS) licus on chromosome 18 affects tumor response and survival in individuals heterozygous for a 28-bp polymorphism in the TS gene. Clin Cancer Res 10:433-439

Wang X, Wang Y, Wang Y, Cheng J, Wang Y, Ha M (2013) Association of thymidylate synthase gene $3^{\prime}$-untranslated region polymorphism with sensitivity of non-small cell lung cancer to pemetrexed treatment: TS gene polymorphism and pemetrexed sensitivity in NSCLC. J Biomed Sci 25(20):5

Wang L, Wang R, Pan Y, Sun Y, Zhang J, Chen H (2014) The pemetrexed-containing treatments in the non-small cell lung cancer, is -/low thymidylate synthase expression better than $+/$ high thymidylate synthase expression: a meta-analysis. BMC Cancer 19(14):205

Zheng Z, Chen T, Li X, Haura E, Sharma A, Bepler G (2007) DNA synthesis and repair genes RRM1 and ERCC1 in lung cancer. N Engl J Med 356(8):800-808 